\title{
Suppression of two-stream hose instabilities at wavelengths shorter than the beam's transverse size
}

\author{
R. A. Bosch \\ Synchrotron Radiation Center, University of Wisconsin-Madison, \\ 3731 Schneider Drive, Stoughton, Wisconsin 53589, USA
}

(Received 25 February 2003; published 7 July 2003)

\begin{abstract}
Transverse hose instability may disrupt the propagation of a charged-particle beam in a channel of oppositely charged particles. A theoretical model predicts stabilization of this two-stream instability when the instability wavelength becomes smaller than the beam's transverse size in a frame of reference where the instability's phase velocity is nonrelativistic. Suppression of short-wavelength instability is also predicted when a proton beam propagates through a channel consisting of electrons and positive ions, consistent with previous experimental results.
\end{abstract}

DOI: 10.1103/PhysRevSTAB.6.074201

PACS numbers: 29.27.Bd, 41.75.-i, 52.35.-g, 52.40.Mj

\section{INTRODUCTION}

When a beam of charged particles propagates in a channel of particles with the opposite charge, a transverse dipole (hose) instability may result [1,2]. For a proton beam, this instability has been called the $e-p$ instability [3-8], while for an electron beam it has been called the ion hose instability [9-13]. For colliding beams, this instability may give rise to the coherent dipole beambeam effect [14]. The instability can adversely affect a magnetically focused beam or a beam whose only focusing is provided by the charged-particle channel.

In this paper, the hose instability is considered theoretically for a flat beam, whose width is much greater than height. We study a rigid-beam model in which damping terms represent the phase-mix damping of beam and channel oscillations. In this model, the force on the beam from a channel perturbation and the force on the channel from a beam perturbation are both reduced for short-wavelength perturbations. As a result, instability is suppressed when the instability wavelength becomes shorter than the beam height in a frame of reference where the instability's phase velocity is nonrelativistic. Typically, this is satisfied in the laboratory frame for the ion hose instability, and in the beam frame for the $e-p$ instability. For the ion hose instability, suppression may occur at a high channel density, while for the $e-p$ instability suppression may occur at a high beam density. For the coherent dipole beam-beam effect, short-wavelength suppression may occur for large values of the disruption parameter [14], where multiple hose oscillations occur within a single bunch.

Our model also predicts instability suppression at wavelengths shorter than the beam height for a proton beam propagating in a channel of electrons and positive ions. This is consistent with previous experiments that demonstrated stable propagation of an intense $1 \mathrm{MeV}$ proton beam in such a channel [15-18].

\section{RIGID-BEAM MODEL OF BEAM AND CHANNEL}

Consider a flat uniform-density beam of height $h$ coasting through a uniform-density oppositely chargedparticle channel with the same height. Vertical centroid perturbations with wavelength $\gg h$ may be approximated by a rigid-beam model with smooth quadrupole focusing and damping terms $[2,10,11]$

$$
\begin{aligned}
\frac{d^{2} b}{d t^{2}}+\omega_{\beta}^{2} b+2 \alpha_{b} \frac{d b}{d t} & =-\omega_{b}^{2}(b-c), \\
\frac{d^{2} c}{d t^{2}}+2 \alpha_{c} \frac{d c}{d t} & =-\omega_{c}^{2}(c-b),
\end{aligned}
$$

where $b(z, t)$ is the beam displacement and $c(z, t)$ is the channel displacement, both functions of propagation distance $z$ and time $t$. Here, $\omega_{\beta}$ is the betatron frequency from smooth magnetic focusing. The "bounce" frequencies of the beam and channel are given by

$$
\omega_{b}=\left(n_{c} e^{2} / \varepsilon_{0} \gamma m_{b}\right)^{1 / 2}, \quad \omega_{c}=\left(n_{b} e^{2} / \varepsilon_{0} m_{c}\right)^{1 / 2},
$$

where $n_{b}$ and $n_{c}$ are the densities of the beam and channel, $m_{b}$ and $m_{c}$ are the masses of the beam and channel particles, $e<0$ is the electron charge, $\varepsilon_{0}$ is the permittivity of free space, and $\gamma$ is the beam's relativistic factor.

Realistic growth rates may be obtained with a rigidbeam model, provided that phase-mix damping of the beam and channel is included [11]. The phase-mix damping may be modeled by considering a distribution of bounce frequencies [9], or more simply by damping terms $[2,5,7,10,11]$. In Eq. (1), $\alpha_{b}$ and $\alpha_{c}$ are the damping rates for beam and channel oscillations. When phase-mix damping is the dominant damping mechanism, reasonable values of these rates give damping within a few oscillations for the case where $\omega_{\beta}=0$ [11]. We therefore consider the values 


$$
\alpha_{b} / \omega_{b}=\alpha_{c} / \omega_{c}=0.1
$$

which are consistent with numerical simulations of the ion hose instability [19] and in approximate agreement with numerical studies of the $e$ - $p$ instability [8]. A larger damping rate $\left(\alpha_{c} / \omega_{c} \approx 0.25\right)$ may be used to model a beam with a large variation in dimensions [20]. For a Lorentzian distribution of bounce frequencies (also called a Cauchy distribution), damping terms describe the phase-mix damping of periodic perturbations whose amplitude is independent of bounce frequency [7,19]. This suggests that damping terms will approximately describe the damping for more realistic bounce-frequency distributions.

For a channel perturbation whose wavelength is comparable to or smaller than the beam height, the electric force upon the beam caused by the channel perturbation is modified by a factor $F\left(h / \lambda_{0}\right)$, whose value is obtained in Appendix A:

$$
F\left(h / \lambda_{0}\right)=\frac{1-\exp \left(-2 \pi h / \lambda_{0}\right)}{\left(2 \pi h / \lambda_{0}\right)} .
$$

The force upon the channel from a beam perturbation is modified by the same factor. Here, $\lambda_{0}$ is the wavelength evaluated in a frame of reference where the perturbation's phase velocity is nonrelativistic, i.e., much smaller than the speed of light. Typically, this is satisfied in the laboratory frame for the ion hose instability, and in the beam frame for the $e-p$ instability. Therefore, a single-wavelength perturbation of arbitrary wavelength may be described by

$$
\Omega^{2}-\left(\omega_{\beta}^{2}+\omega_{b}^{2}\right)+2 i \alpha_{b} \Omega=F^{2} \omega_{b}^{2} \omega_{c}^{2} /\left[\left(\Omega+m \omega_{0}\right)^{2}-\omega_{c}^{2}+2 i \alpha_{c}\left(\Omega+m \omega_{0}\right)\right],
$$

where $\omega_{0}=v / R$ is the beam's angular revolution frequency.

In a linear accelerator, a disturbance of any wavelength is possible, a worst-case scenario. An oscillation with real frequency $\omega$ may be characterized by solving Eq. (7) for complex $k(\omega)$. This describes the case of a "tickler" excitation at frequency $\omega[19]$. The resulting dispersion relation is

$$
v k=\omega+i \alpha_{b} \pm\left[\omega_{\beta}^{2}+\omega_{b}^{2}-\alpha_{b}^{2}+\frac{F^{2} \omega_{b}^{2} \omega_{c}^{2}}{\omega^{2}-\omega_{c}^{2}+2 i \alpha_{c} \omega}\right]^{1 / 2}
$$

For brevity, we consider a linear accelerator with real frequency $\omega$ in this article. A strong-focusing circular accelerator is considered in Appendix B to show that short-wavelength instability suppression also occurs in circular geometry.

The modes where $\omega \operatorname{Re}(k)>0$ and $\operatorname{Im}(k)<0$ describe hose oscillations that propagate downstream and grow, with laboratory-frame wavelength $\lambda=|2 \pi / \operatorname{Re}(k)|$ and spatial growth rate $-\operatorname{Im}(k)$. The long-term growth of a disturbance is dominated by the frequency with maximum growth, which very nearly equals the channel bounce frequency. For this case of resonant excitation $\left(\omega=\omega_{c}\right)$, the instability wavelength and spatial growth rate obey

$$
\begin{aligned}
v k= & \omega_{c}+i \alpha_{b} \\
& \pm\left[\omega_{\beta}^{2}+\omega_{b}^{2}-\alpha_{b}^{2}-i\left(F^{2} \omega_{b}^{2} \omega_{c} / 2 \alpha_{c}\right)\right]^{1 / 2} .
\end{aligned}
$$

The approximate validity of Eqs. (9) and (10) is supported by application to the ion hose instability of an electron beam propagating in an ion channel with $\omega_{b} \gg \omega_{\beta}, \omega_{b} \gg \omega_{c}$, and $\lambda_{0} \gg h$. For this case [11], the instability wavelength, growth rate, rise time, and group velocity from Eqs. (9) and (10) are in approximate 
agreement with numerical modeling [9] and experiments $[12,13]$.

\section{A. Strong magnetic focusing}

When magnetic focusing of the beam greatly exceeds focusing by the channel $\left(\omega_{\beta} \gg \omega_{b}\right)$, the most unstable solution of Eq. (10) is a "backward" wave, whose phase velocity is smaller than the beam velocity

$$
\begin{aligned}
v k \approx & \omega_{c}+\omega_{\beta}+\omega_{b}^{2} / 2 \omega_{\beta}-\alpha_{b}^{2} / 2 \omega_{\beta} \\
& +i\left(\alpha_{b}-F^{2} \omega_{b}^{2} \omega_{c} / 4 \alpha_{c} \omega_{\beta}\right) .
\end{aligned}
$$

For a typical ion hose instability with $\omega_{\beta} \gg \omega_{c}$, the instability wavelength is determined by the betatron frequency from magnetic focusing, and the phase velocity $\omega / \operatorname{Re}(k)$ is nonrelativistic in the laboratory frame. For a typical $e-p$ instability where $\omega_{c} \gg \gamma^{2} \omega_{\beta}$ is obeyed [4-8], the instability wavelength is determined by the bounce frequency of the electron channel, and the phase velocity is nonrelativistic in the beam frame [i.e., $\mid v-$ $\omega / \operatorname{Re}(k) \mid \ll c_{l} / \gamma^{2}$, where $c_{l}$ is the speed of light].

Equation (11) predicts stability $[\operatorname{Im}(k)>0]$ when the incoherent betatron-frequency shift from the channel $\Delta \omega_{\beta}$ satisfies

$$
\frac{\Delta \omega_{\beta}}{\omega_{\beta}} \approx \frac{\omega_{b}^{2}}{2 \omega_{\beta}^{2}}<\frac{8 \alpha_{b}^{2} \alpha_{c}^{2}}{F^{4} \omega_{b}^{2} \omega_{c}^{2}} .
$$

When phase-mix damping described by Eq. (3) is the dominant damping mechanism, this condition for stability becomes

$$
\frac{\Delta \omega_{\beta}}{\omega_{\beta}}<\frac{0.0008}{F^{4}} .
$$

For a long-wavelength perturbation with $\lambda_{0} \gg h$, the factor $F \approx 1$, so that stability requires that $\Delta \omega_{\beta} / \omega_{\beta}<$ 0.0008 . When $\lambda_{0}<h, F$ becomes less than 0.16 and instability is suppressed throughout the strong magnetic focusing regime (where $\omega_{\beta} \gg \omega_{b}$ ). For a typical ion hose instability with $\omega_{\beta} \gg \omega_{c}$, suppression is therefore predicted when the laboratory wavelength $\lambda=\lambda_{0}$ is smaller than the beam height $h$. For a typical $e-p$ instability with $\omega_{c} \gg \gamma^{2} \omega_{\beta}$, suppression is predicted when the beamframe wavelength $\lambda_{0} \approx \gamma \lambda$ is smaller than $h$.

\section{B. Weak magnetic focusing}

Let us now consider the case where focusing by the channel dominates $\left(\omega_{b} \gg \omega_{\beta}\right)$. Anticipating that instability suppression requires a small value of $F$, we consider Eq. (10) for $F^{2} \omega_{c} / 2 \alpha_{c} \ll 1$. The most unstable solution is a backward wave that obeys

$$
v k \approx \omega_{c}+\omega_{b}+i\left(\alpha_{b}-F^{2} \omega_{b} \omega_{c} / 4 \alpha_{c}\right) .
$$

For a typical ion hose instability with $\omega_{b} \gg \omega_{c}[12,13]$, the instability wavelength is determined by the beam's bounce frequency in the ion channel, and the phase velocity is nonrelativistic in the laboratory frame. For a typical $e-p$ instability with $\omega_{c} \gg \gamma^{2} \omega_{b}$, the instability wavelength is determined by the bounce frequency of the electron channel, and the phase velocity is nonrelativistic in the beam frame.

Equation (14) predicts stability when

$$
F\left(h / \lambda_{0}\right)<2\left(\frac{\alpha_{b} \alpha_{c}}{\omega_{b} \omega_{c}}\right)^{1 / 2} .
$$

For the case where phase-mix damping described by Eq. (3) is the dominant damping mechanism, this condition for stability becomes

$$
F\left(h / \lambda_{0}\right)<0.2 \text {, }
$$

which occurs when $\lambda_{0}<1.25 h$. For a typical ion hose instability, suppression is predicted when the laboratory wavelength $\lambda=\lambda_{0}<1.25 h$. For a typical $e-p$ instability, suppression is predicted when the beam-frame wavelength $\lambda_{0} \approx \gamma \lambda<1.25 h$.

\section{THE $e-p$ INSTABILITY WITH THREE SPECIES}

In experiments where the short-wavelength $e$ - $p$ instability was apparently suppressed [15-18], a $1 \mathrm{MeV}$ proton beam propagated through a channel of electrons containing a secondary species of positive ions. Let $s(z, t)$ denote the centroid position of a secondary species with density $n_{s}$ and mass $m_{s} \gg m_{c}$, whose charge is the same as the beam. We generalize Eq. (5) to

$$
\begin{aligned}
\frac{d^{2} b}{d t^{2}}+\omega_{\beta}^{2} b+2 \alpha_{b} \frac{d b}{d t} & =-\omega_{b}^{2}(b-F c)+\omega_{b s}^{2}(b-F s), \\
\frac{d^{2} c}{d t^{2}}+2 \alpha_{c} \frac{d c}{d t} & =-\omega_{c}^{2}(c-F b)-\omega_{c s}^{2}(c-F s), \\
\frac{d^{2} s}{d t^{2}}+2 \alpha_{s} \frac{d s}{d t} & =\omega_{s b}^{2}(s-F b)-\omega_{s c}^{2}(s-F c),
\end{aligned}
$$

where

$$
\begin{aligned}
& \omega_{b s}=\left(n_{s} e^{2} / \varepsilon_{0} \gamma m_{b}\right)^{1 / 2}, \quad \omega_{s b}=\left(n_{b} e^{2} / \varepsilon_{0} m_{s}\right)^{1 / 2}, \\
& \omega_{c s}=\left(n_{s} e^{2} / \varepsilon_{0} m_{c}\right)^{1 / 2}, \quad \omega_{s c}=\left(n_{c} e^{2} / \varepsilon_{0} m_{s}\right)^{1 / 2} .
\end{aligned}
$$

Focusing of the beam by the channel and secondaries requires that $\omega_{b}>\omega_{b s}$ (i.e., $n_{c}>n_{s}$ ). When the dominant damping mechanism for the beam and channel is phase-mix damping within a few oscillation periods, the damping rates approximately obey

$$
\frac{\alpha_{b}}{\left(\omega_{b}^{2}-\omega_{b s}^{2}\right)^{1 / 2}}=\frac{\alpha_{c}}{\left(\omega_{c}^{2}+\omega_{c s}^{2}\right)^{1 / 2}}=0.1 .
$$

Larger damping rates may be used to model a beam with a large variation in dimensions. 
For a disturbance of the form $b(z, t)=b_{0} \exp [i(k z-\omega t)]$, Eq. (17) gives the dispersion relation:

$$
\begin{aligned}
v k=\omega & +i \alpha_{b} \\
\pm & {\left[-\alpha_{b}^{2}+\omega_{\beta}^{2}+\omega_{b}^{2}-\omega_{b s}^{2}+\frac{F^{2} \omega_{b s}^{2} \omega_{s b}^{2}}{\omega^{2}+2 i \alpha_{s} \omega+\omega_{s b}^{2}-\omega_{s c}^{2}}\right.} \\
& \left.+\frac{\left(F \omega_{b}^{2}+\frac{F^{2} \omega_{b s}^{2} \omega_{s c}^{2}}{\omega^{2}+2 i \alpha_{s} \omega+\omega_{s b}^{2}-\omega_{s c}^{2}}\right)\left(F \omega_{c}^{2}+\frac{F^{2} \omega_{c s}^{2} \omega_{s b}^{2}}{\omega^{2}+2 i \alpha_{s} \omega+\omega_{s b}^{2}-\omega_{s c}^{2}}\right)}{\omega^{2}+2 i \alpha_{c} \omega-\omega_{c}^{2}-\omega_{c s}^{2}-\frac{F^{2} \omega_{c s}^{2} \omega_{s c}^{2}}{\omega^{2}+2 i \alpha_{s} \omega+\omega_{s b}^{2}-\omega_{s c}^{2}}}\right]^{1 / 2} .
\end{aligned}
$$

For resonant excitation of the channel $\left(\omega^{2}=\omega_{c}^{2}+\omega_{c s}^{2}\right.$ with $\left.\omega>0\right)$, Eq. (20) describes the three-species $e-p$ instability:

$$
\begin{aligned}
v k=\left(\omega_{c}^{2}+\omega_{c s}^{2}\right)^{1 / 2}+i \alpha_{b} & \pm\left[-\alpha_{b}^{2}+\omega_{\beta}^{2}+\omega_{b}^{2}-\omega_{b s}^{2}+\frac{F^{2} \omega_{b s}^{2} \omega_{s b}^{2}}{\omega_{c}^{2}+\omega_{c s}^{2}+\omega_{s b}^{2}-\omega_{s c}^{2}+2 i \alpha_{s} \omega}\right. \\
& \left.+\frac{\left(F \omega_{b}^{2}+\frac{F^{2} \omega_{b s}^{2} \omega_{s c}^{2}}{\omega_{c}^{2}+\omega_{c s}^{2}+\omega_{s b}^{2}-\omega_{s c}^{2}+2 i \alpha_{s} \omega}\right)\left(F \omega_{c}^{2}+\frac{F^{2} \omega_{c s}^{2} \omega_{s b}^{2}}{\omega_{c}^{2}+\omega_{c s}^{2}+\omega_{s b}^{2}-\omega_{s c}^{2}+2 i \alpha_{s} \omega}\right)}{2 i \alpha_{c} \omega-\frac{F^{2} \omega_{c s}^{2} \omega_{s c}^{2}}{\omega_{c}^{2}+\omega_{c s}^{2}+\omega_{s b}^{2}-\omega_{s c}^{2}+2 i \alpha_{s} \omega}}\right]^{1 / 2}
\end{aligned}
$$

Because of the small electron mass, $\omega^{2}=\omega_{c}^{2}+\omega_{c s}^{2} \gg \omega_{s c}^{2}$ is typically obeyed [16]. For example, this relation is satisfied in the quasineutral case where $n_{b}+n_{s} \approx n_{c}$. Consequently, Eq. (21) simplifies to

$$
v k \approx\left(\omega_{c}^{2}+\omega_{c s}^{2}\right)^{1 / 2}+i \alpha_{b} \pm\left[-\alpha_{b}^{2}+\omega_{\beta}^{2}+\omega_{b}^{2}-\omega_{b s}^{2}-\frac{i F^{2} \omega_{b}^{2} \omega_{c}^{2}}{2 \alpha_{c}\left(\omega_{c}^{2}+\omega_{c s}^{2}\right)^{1 / 2}}\right]^{1 / 2} .
$$

\section{A. Strong magnetic focusing}

For strong magnetic focusing $\left(\omega_{\beta}^{2} \gg \omega_{b}^{2}-\omega_{b s}^{2}\right)$, the most unstable solution to Eq. (22) is

$$
v k \approx\left(\omega_{c}^{2}+\omega_{c s}^{2}\right)^{1 / 2}+\omega_{\beta}+\frac{\omega_{b}^{2}}{2 \omega_{\beta}}-\frac{\alpha_{b}^{2}}{2 \omega_{\beta}}-\frac{\omega_{b s}^{2}}{2 \omega_{\beta}}+i\left[\alpha_{b}-\frac{F^{2} \omega_{b}^{2} \omega_{c}^{2}}{4 \alpha_{c}\left(\omega_{c}^{2}+\omega_{c s}^{2}\right)^{1 / 2} \omega_{\beta}}\right] .
$$

For a typical $e-p$ instability where $\left(\omega_{c}^{2}+\omega_{c s}^{2}\right)^{1 / 2} \gg \gamma^{2} \omega_{\beta}$ is obeyed, the instability wavelength is determined by the natural frequency of channel oscillations $\left(\omega_{c}^{2}+\omega_{c s}^{2}\right)^{1 / 2}$, and the phase velocity is nonrelativistic in the beam frame. Equation (23) predicts stability when the incoherent betatron-frequency shift from the channel and secondaries obeys

$$
\begin{aligned}
\frac{\Delta \omega_{\beta}}{\omega_{\beta}} \approx & \frac{\omega_{b}^{2}-\omega_{b s}^{2}}{2 \omega_{\beta}^{2}} \\
& <\frac{8}{F^{4}}\left(\frac{\alpha_{b}^{2}}{\omega_{b}^{2}-\omega_{b s}^{2}}\right)\left(\frac{\alpha_{c}^{2}}{\omega_{c}^{2}+\omega_{c s}^{2}}\right)\left(\frac{\omega_{b}^{2}-\omega_{b s}^{2}}{\omega_{b}^{2}}\right)^{2}\left(\frac{\omega_{c}^{2}+\omega_{c s}^{2}}{\omega_{c}^{2}}\right)^{2} \\
= & \frac{8}{F^{4}}\left(\frac{\alpha_{b}^{2}}{\omega_{b}^{2}-\omega_{b s}^{2}}\right)\left(\frac{\alpha_{c}^{2}}{\omega_{c}^{2}+\omega_{c s}^{2}}\right)\left[1-\frac{n_{s}}{n_{b} n_{c}}\left(n_{b}+n_{s}-n_{c}\right)\right]^{2}
\end{aligned}
$$

For the case where phase-mix damping described by Eq. (19) is the dominant damping mechanism, this condition for stability becomes

$$
\frac{\Delta \omega_{\beta}}{\omega_{\beta}}<\frac{0.0008}{F^{4}}\left[1-\frac{n_{s}}{n_{b} n_{c}}\left(n_{b}+n_{s}-n_{c}\right)\right]^{2} .
$$

When the beam's space charge is neutralized by the channel and secondaries $\left(n_{b}+n_{s}-n_{c}=0\right)$, the RHS of Eq. (25) equals the RHS of Eq. (13). For long wavelengths with $\lambda_{0} \gg h$, the factor $F \approx 1$, so that stability requires that $\Delta \omega_{\beta} / \omega_{\beta}<0.0008$. When the beam-frame instability wavelength becomes shorter than the beam height $\left(\lambda_{0} \approx \gamma \lambda<h\right), F$ becomes less than 0.16 and instability is suppressed throughout the strong magnetic focusing regime (where $\omega_{\beta}^{2} \gg \omega_{b}^{2}-\omega_{b s}^{2}$ ).

\section{B. Weak magnetic focusing}

Consider weak magnetic focusing with $\omega_{\beta}^{2} \ll \omega_{b}^{2}-$ $\omega_{b s}^{2}$. Provided that $F^{2} \omega_{c}^{2} \omega_{b}^{2} / 2 \alpha_{c}\left(\omega_{c}^{2}+\omega_{c s}^{2}\right)^{1 / 2}\left(\omega_{b}^{2}-\right.$ $\left.\omega_{b s}^{2}\right) \ll 1$, the most unstable solution of Eq. (22) is

$$
\begin{aligned}
v k \approx & \left(\omega_{c}^{2}+\omega_{c s}^{2}\right)^{1 / 2}+\left(\omega_{b}^{2}-\omega_{b s}^{2}\right)^{1 / 2} \\
& +i\left[\alpha_{b}-\frac{F^{2} \omega_{b}^{2} \omega_{c}^{2}}{4 \alpha_{c}\left(\omega_{c}^{2}+\omega_{c s}^{2}\right)^{1 / 2}\left(\omega_{b}^{2}-\omega_{b s}^{2}\right)^{1 / 2}}\right] .
\end{aligned}
$$


For a typical $e-p$ instability where $\left(\omega_{c}^{2}+\omega_{c s}^{2}\right)^{1 / 2} \gg \gamma^{2}\left(\omega_{b}^{2}-\omega_{b s}^{2}\right)^{1 / 2}$ is obeyed, the instability wavelength is determined by the natural frequency of channel oscillations $\left(\omega_{c}^{2}+\omega_{c s}^{2}\right)^{1 / 2}$, and the phase velocity is nonrelativistic in the beam frame. Equation (26) predicts stability for

$$
\begin{aligned}
F & <2\left[\frac{\alpha_{b} \alpha_{c}}{\left(\omega_{b}^{2}-\omega_{b s}^{2}\right)^{1 / 2}\left(\omega_{c}^{2}+\omega_{c s}^{2}\right)^{1 / 2}}\right]^{1 / 2}\left(\frac{\omega_{b}^{2}-\omega_{b s}^{2}}{\omega_{b}^{2}}\right)^{1 / 2}\left(\frac{\omega_{c}^{2}+\omega_{c s}^{2}}{\omega_{c}^{2}}\right)^{1 / 2} \\
& =2\left[\frac{\alpha_{b} \alpha_{c}}{\left(\omega_{b}^{2}-\omega_{b s}^{2}\right)^{1 / 2}\left(\omega_{c}^{2}+\omega_{c s}^{2}\right)^{1 / 2}}\right]^{1 / 2}\left[1-\frac{n_{s}}{n_{b} n_{c}}\left(n_{b}+n_{s}-n_{c}\right)\right]^{1 / 2} .
\end{aligned}
$$

For the case where the dominant source of damping is phase-mix damping with the rate given by Eq. (19), this condition for stability becomes

$$
F<0.2\left[1-\frac{n_{s}}{n_{b} n_{c}}\left(n_{b}+n_{s}-n_{c}\right)\right]^{1 / 2} .
$$

When the beam's space charge is neutralized by the channel and secondaries $\left(n_{b}+n_{s}-n_{c}=0\right), \quad F<0.2$ gives stability. Thus, instability suppression is predicted when the instability wavelength in the beam frame $\lambda_{0} \approx$ $\gamma \lambda$ becomes shorter than $1.25 h$.

When propagation of a proton beam through a plasma channel results in space-charge neutrality, a sufficiently dense plasma may shorten the $e-p$ instability wavelength in the laboratory below $1.25 h / \gamma$, thereby suppressing the instability.

\section{DISCUSSION}

A charged-particle beam propagating in a channel of oppositely charged particles may undergo a transverse hose instability. For a flat coasting beam of uniform density, this instability was considered by using a rigidbeam model with damping terms that approximate phasemix damping. For short-wavelength perturbations, the transverse force upon the beam from a channel perturbation and the transverse force on the channel from a beam perturbation are reduced. As a result, our model predicts suppression of the hose instability when the instability wavelength becomes shorter than the beam height in a frame of reference where the instability's phase velocity is nonrelativistic. Typically, this is satisfied in the laboratory frame for the ion hose instability, and in the beam frame for the $e-p$ instability. For the ion hose instability, suppression may occur at a high channel density, while for the $e-p$ instability suppression may occur at a high beam density. For the coherent dipole beam-beam effect, suppression may occur for large values of the disruption parameter.

The suppression of short-wavelength hose instability is also predicted when the space charge of a proton beam is neutralized by a channel of electrons and positive ions (a plasma channel). This prediction agrees with the interpretation of experiments in which the $e-p$ instability of a $1 \mathrm{MeV}$ proton beam was suppressed by introducing secondary ions [15-18].

However, a rigid-beam model is a simplification that does not include changes in the shape or size of the beam and channel, or longitudinal forces. Thus, studying hose instabilities at short wavelengths with simulations or additional experiments is necessary to verify this prediction. It should also be noted that suppression of hose instability does not ensure successful beam propagation, since additional transverse modes or longitudinal modes may disrupt the beam.

\section{ACKNOWLEDGMENTS}

The author appreciates valuable discussions with K. D. Jacobs and J. J. Bisognano. This work is based upon research conducted at the Synchrotron Radiation Center, University of Wisconsin-Madison, which is supported by the National Science Foundation under Award No. DMR0084402.

\section{APPENDIX A: ELECTRICAL ATTRACTION BETWEEN FLAT BEAM AND CHANNEL}

For a uniform beam and channel of infinite width and height $h$, consider the force exerted upon the beam by a channel for a perturbation of wavelength $\lambda_{0}$. For a typical ion hose instability, the phase velocity of the perturbation is nonrelativistic in the laboratory, so that the electric field in the laboratory frame may be obtained in the electrostatic approximation. We consider a channel whose particles have charge $-e$ with the centroid position given by $c(z)=c_{0} \cos k_{0} z$ where $k_{0} \equiv 2 \pi / \lambda_{0}>0$. The channel density is

$$
n(y, z)=n_{c}, \quad \text { for }\left|y-c_{0} \cos k_{0} z\right|<h / 2 .
$$

In two dimensions, $\nabla^{2} \ln \left|\boldsymbol{r}-\boldsymbol{r}^{\prime}\right|=2 \pi \delta\left(\boldsymbol{r}-\boldsymbol{r}^{\prime}\right)$, so that the channel's electrostatic potential $\phi$ (which obeys $\left.\nabla^{2} \phi=n e / \varepsilon_{0}\right)$ is given by

$$
\begin{aligned}
\phi(y, z) & =\frac{e}{2 \pi \varepsilon_{0}} \int_{-\infty}^{\infty} d z^{\prime} \int_{-\infty}^{\infty} d y^{\prime} n\left(y^{\prime}, z^{\prime}\right) \ln \sqrt{\left(y-y^{\prime}\right)^{2}+\left(z-z^{\prime}\right)^{2}} \\
& =\frac{e n_{c}}{4 \pi \varepsilon_{0}} \int_{-\infty}^{\infty} d z^{\prime} \int_{-h / 2+c_{0} \cos k_{0} z^{\prime}}^{h / 2+c_{0} \cos k_{0} z^{\prime}} d y^{\prime} \ln \left[\left(y-y^{\prime}\right)^{2}+\left(z-z^{\prime}\right)^{2}\right] .
\end{aligned}
$$

The transverse electric field from the channel is therefore 


$$
E_{y}(y, z)=-\frac{\partial \phi}{\partial y}=\frac{e n_{c}}{4 \pi \varepsilon_{0}} \int_{-\infty}^{\infty} d z^{\prime} \int_{-h / 2+c_{0} \cos k_{0} z^{\prime}}^{h / 2+c_{0} \cos k_{0} z^{\prime}} d y^{\prime} \frac{2\left(y^{\prime}-y\right)}{\left(y-y^{\prime}\right)^{2}+\left(z-z^{\prime}\right)^{2}}
$$

Substituting $y^{\prime \prime}=y^{\prime}-y$ and integrating over $y^{\prime \prime}$ gives

$$
E_{y}(y, z)=\frac{e n_{c}}{4 \pi \varepsilon_{0}} \int_{-\infty}^{\infty} d z^{\prime}\left\{\ln \left[\left(h / 2+c_{0} \cos k_{0} z^{\prime}-y\right)^{2}+\left(z-z^{\prime}\right)^{2}\right]-\ln \left[\left(-h / 2+c_{0} \cos k_{0} z^{\prime}-y\right)^{2}+\left(z-z^{\prime}\right)^{2}\right]\right\} .
$$

Substituting $z^{\prime \prime}=z^{\prime}-z$ and expanding for a small perturbation $c_{0}$ gives

$$
\begin{aligned}
& E_{y}(y, z)=\frac{e n_{c}}{4 \pi \varepsilon_{0}} \int_{-\infty}^{\infty} d z^{\prime \prime}\left\{\ln \left[(h / 2-y)^{2}+2(h / 2-y) c_{0} \cos k_{0}\left(z^{\prime \prime}+z\right)+z^{\prime \prime 2}\right]\right. \\
&\left.-\ln \left[(h / 2+y)^{2}-2(h / 2+y) c_{0} \cos k_{0}\left(z^{\prime \prime}+z\right)+z^{\prime \prime 2}\right]\right\} \\
& \approx \frac{e n_{c}}{4 \pi \varepsilon_{0}} \int_{-\infty}^{\infty} d z^{\prime \prime}\left\{\begin{array}{c}
\ln \left[(h / 2-y)^{2}+z^{\prime \prime 2}\right]+\frac{2(h / 2-y) c_{0} \cos k_{0}\left(z^{\prime \prime}+z\right)}{(h / 2-y)^{2}+z^{\prime \prime 2}} \\
\left.-\ln \left[(h / 2+y)^{2}+z^{\prime \prime 2}\right]+\frac{2(h / 2+y) c_{0} \cos k_{0}\left(z^{\prime \prime}+z\right)}{(h / 2+y)^{2}+z^{\prime \prime 2}}\right\}
\end{array}\right\}
\end{aligned}
$$

Using the relations $\int d x \ln \left(x^{2}+a^{2}\right)=x \ln \left(x^{2}+a^{2}\right)-2 x+2 a \tan ^{-1}(x / a)$ and $\int_{0}^{\infty} d x \cos \alpha x /\left(x^{2}+\beta^{2}\right)=$ $(\pi / 2 \beta) \exp (-\alpha \beta)$ for $\alpha \geq 0, \beta>0$ [22], we obtain

$$
\begin{aligned}
E_{y}(y, z)= & \frac{e n_{c}}{2 \varepsilon_{0}}(|h / 2-y|-|h / 2+y|) \\
& +\frac{e n_{c}}{2 \varepsilon_{0}}\left(c_{0} \cos k_{0} z\right)\left[\frac{h / 2-y}{|h / 2-y|} \exp \left(-k_{0}|h / 2-y|\right)+\frac{h / 2+y}{|h / 2+y|} \exp \left(-k_{0}|h / 2+y|\right)\right] .
\end{aligned}
$$

The average transverse field acting upon the beam at longitudinal position $z$ depends upon the beam's centroid position $b(z)$

$$
\left\langle E_{y}\right\rangle=\frac{1}{h} \int_{-h / 2+b(z)}^{h / 2+b(z)} d y E_{y}(y, z)
$$

To lowest order in $b(z)$ and $c(z)$, Eqs. (A6) and (A7) give

$$
\left\langle E_{y}\right\rangle=\frac{-e n_{c}}{\varepsilon_{0}} b(z)+\frac{e n_{c}}{\varepsilon_{0}}\left[\frac{1-\exp \left(-k_{0} h\right)}{k_{0} h}\right] c_{0} \cos k_{0} z=\frac{-e n_{c}}{\varepsilon_{0}}\left[b(z)-F\left(h / \lambda_{0}\right) c(z)\right],
$$

where

$$
F\left(h / \lambda_{0}\right)=\frac{1-\exp \left(-2 \pi h / \lambda_{0}\right)}{\left(2 \pi h / \lambda_{0}\right)} .
$$

On the RHS of Eq. (A8), the first team equals the average electric field on the displaced beam segment from an unperturbed channel, while the second term equals the average electric field experienced by an unperturbed beam segment from the periodic channel perturbation. The first term is independent of the wavelength of the perturbation while the second term is proportional to a factor $F\left(h / \lambda_{0}\right)$. For a perturbation whose wavelength is comparable to or smaller than the beam height, the factor $F$ reduces the force on the beam caused by the channel perturbation $c(z)$. Multiplying the average transverse field by $e / m_{b} \gamma$, we find that the contribution to the beam's acceleration from the channel is $-\omega_{b}^{2}(b-F c)$.

To determine the average transverse field on the channel from a beam whose particles have charge $e$ with centroid position $b(z)=b_{0} \cos k_{0} z$, we substitute

$b \rightarrow c, \quad c \rightarrow b, \quad$ and $e \rightarrow-e$ in Eqs. (A1)-(A9). Consequently, the contribution to the channel's acceleration from a perturbed beam is given by $-\omega_{c}^{2}(c-F b)$. The same factor $F\left(h / \lambda_{0}\right)$ modifies the acceleration of the channel by a beam perturbation and the acceleration of the beam by a channel perturbation.

For a typical $e-p$ instability of a relativistic beam, the phase velocity of the perturbation is relativistic in the laboratory frame and nonrelativistic in the beam frame [i.e., $|v-\operatorname{Re}(\omega) / \operatorname{Re}(k)| \ll c_{l} / \gamma^{2}$, where $c_{l}$ is the speed of light]. Consequently, the electrostatic approximation is valid in the beam frame, but not in the laboratory. In this case, Eqs. (A1)-(A9) apply in the beam frame, and the wavelength $\lambda_{0}$ in Eq. (A9) is the wavelength of the perturbation evaluated in the beam frame, which is related to the laboratory-frame wavelength by $\lambda_{0} \approx \gamma \lambda$.

For either a typical ion hose instability or typical $e-p$ instability, $\lambda_{0}=2 \pi / k_{0}$, where $k_{0}$ is the real wave number in a frame of reference where the real frequency is zero. 
Since the real frequency and wave number form a 4vector [21], $k_{0}^{2}=[\operatorname{Re}(k)]^{2}-[\operatorname{Re}(\omega)]^{2} / c_{l}^{2}$.

The factor $F$ given by Eq. (A9) is valid for a uniformdensity beam and channel with width $\gg$ height. Expressions for the electric field of a round beam with a dipole perturbation [23] or periodic density perturbation [24] indicate that a short-wavelength modification of beam-channel forces also occurs with round beams.

\section{APPENDIX B: INSTABILITY SUPPRESSION IN A STORAGE RING}

Consider a circular accelerator with strong magnetic focusing where the beam propagates through a channel of oppositely charged particles. For a perturbed betatron oscillation with $\Omega \approx-\omega_{\beta}$, Eq. (8) yields

$$
\Omega \approx-\omega_{\beta}-\frac{\omega_{b}^{2}}{2 \omega_{\beta}}-i \alpha_{b}-\frac{F^{2} \omega_{b}^{2} \omega_{c}^{2}}{2 \omega_{\beta}\left[\left(-\omega_{\beta}+m \omega_{0}\right)^{2}-\omega_{c}^{2}+2 i \alpha_{c}\left(-\omega_{\beta}+m \omega_{0}\right)\right]} .
$$

Instability growth is largest when there is a betatron sideband whose frequency nearly equals the channel bounce frequency: $-\omega_{\beta}+m \omega_{0} \approx \omega_{c}$. For this case

$$
\Omega \approx-\omega_{\beta}-\frac{\omega_{b}^{2}}{2 \omega_{\beta}}-i\left(\alpha_{b}-\frac{F^{2} \omega_{b}^{2} \omega_{c}}{4 \omega_{\beta} \alpha_{c}}\right) .
$$

Stability occurs when $\operatorname{Im}(\Omega)<0$, i.e., when

$$
\frac{\Delta \omega_{\beta}}{\omega_{\beta}} \approx \frac{\omega_{b}^{2}}{2 \omega_{\beta}^{2}}<\frac{8 \alpha_{b}^{2} \alpha_{c}^{2}}{F^{4} \omega_{b}^{2} \omega_{c}^{2}} .
$$

Equation (B3) is identical to Eq. (12), which is the stability condition for a linear accelerator. Thus, the stability condition for a linear accelerator ensures stability for the worst case in circular geometry-when $-\omega_{\beta}+m \omega_{0} \approx$ $\omega_{c}$ is obeyed for an integral value of $m$.

[1] G. I. Budker, Sov. J. At. En. 1, 673 (1956).

[2] B.V. Chirikov, Plasma Phys. 8, 455 (1966).

[3] G. I. Budker, G. I. Dimov, and V. G. Dudnikov, Sov. J. At. En. 22, 441 (1967).

[4] D. Neuffer, E. Colton, D. Fitzgerald, T. Hardek, R. Hutson, R. Macek, M. Plum, H. Thiessen, and T.-S. Wang, Nucl. Instrum. Methods Phys. Res., Sect. A 321, 1 (1992).

[5] Tai-Sen F. Wang, in Proceedings of the 1995 Particle Accelerator Conference, Dallas (IEEE, Piscataway, NJ, 1996), p. 3143.

[6] H. Qin, R. C. Davidson, and W.W. Lee, Phys. Lett. A 272, 389 (2000).

[7] Tai-Sen F. Wang, P. J. Channell, R. J. Macek, and R. C. Davidson, Phys. Rev. ST Accel. Beams 6, 014204 (2003).

[8] H. Qin, E. A. Startsev, and R. C. Davidson, Phys. Rev. ST Accel. Beams 6, 014401 (2003).

[9] H. L. Buchanon, Phys. Fluids 30, 221 (1987).

[10] R. A. Bosch and R. M. Gilgenbach, Phys. Fluids 31, 634 (1988).
[11] R. A. Bosch and R. M. Gilgenbach, Phys. Fluids 31, 2006 (1988).

[12] R. F. Lucey, Jr., R. M. Gilgenbach, J. D. Miller, J. E. Tucker, and R. A. Bosch, Phys. Fluids B 1, 430 (1989).

[13] R. J. Lipinski, J. R. Smith, I. R. Shokair, K.W. Struve, P. Werner, D. J. Armistead, P. D. Kiekel, I. Molina, and S. Hogeland, Phys. Fluids B 2, 2764 (1990).

[14] R. Li and J. J. Bisognano, Phys. Rev. E 48, 3965 (1993).

[15] G. I. Budker, G. I. Dimov, V. G. Dudnikov, A. A. Sokolov, and V.G. Shamovsky, in Proceedings of the Sixth International Conference on High Energy Accelerators, Cambridge, MA, 1967, edited by R. A. Mack (Cambridge Electron Accelerator Laboratory, Cambridge, MA, 1967), p. 422.

[16] G. I. Dimov and V. E. Chupriyanov, Part. Accel. 14, 155 (1984).

[17] V. Dudnikov, in Proceedings of the 2001 Particle Accelerator Conference, Chicago (IEEE, Piscataway, NJ, 2001), p. 1892.

[18] V. Dudnikov, in High Intensity and High Brightness Hadron Beams, edited by W. Chou, Y. Mori, D. Neuffer, and J.-F. Ostiguy, AIP Conf. Proc. No. 642 (AIP, New York, 2002), p. 367.

[19] G. J. Caporaso and A. G. Cole, in The Physics of Particle Accelerators, edited by M. Month and M. Dienes, AIP Conf. Proc. No. 249 (AIP, New York, 1992), p. 1615.

[20] M. Blaskiewicz, M. A. Furman, M. Pivi, and R. J. Macek, Phys. Rev. ST Accel. Beams 6, 014203 (2003).

[21] J. D. Jackson, Classical Electrodynamics (Wiley, New York, 1975), 2nd ed., p. 521.

[22] I. S. Gradshteyn and I. M. Ryzhik, Table of Integrals, Series, and Products (Academic Press, San Diego, 1994), 5th ed., p. 250, Eq. 2.733.1; p. 445, Eq. 3.723.2.

[23] L. Palumbo and V. G. Vaccaro, in Proceedings of the CERN Accelerator School: Advanced Accelerator Physics, Oxford, England, 1985, edited by S. Turner (CERN, Geneva, 1987), p. 341.

[24] J. D. Miller and R. M. Gilgenbach, Phys. Fluids 30, 3165 (1987). 